\title{
Effect of contralateral augmentation on postoperative complications after the second stage of tissue expander/implant breast reconstruction
}

\author{
Jin-Woo Park ${ }^{1}$, Suhwan Kim², Byung-Joon Jeon ${ }^{2}$, Goo-Hyun Mun ${ }^{2}$, Sa Ik Bang ${ }^{2}$, Jai-Kyong Pyon ${ }^{2}$ \\ ${ }^{1}$ Department of Plastic Surgery, Ewha Womans University Mokdong Hospital, College of Medicine, Ewha Womans University, Seoul, Republic of \\ Korea; ${ }^{2}$ Department of Plastic Surgery, Samsung Medical Center, Sungkyunkwan University School of Medicine, Seoul, Republic of Korea \\ Contributions: (I) Conception and design: JW Park, JK Pyon; (II) Administrative support: JW Park, S Kim; (III) Provision of study materials or \\ patients: BJ Jeon, GH Mun, SI Bang, JK Pyon; (IV) Collection and assembly of data: JW Park, S Kim; (V) Data analysis and interpretation: JW Park, \\ GH Mun, SI Bang, JK Pyon; (VI) Manuscript writing: All authors; (VII) Final approval of manuscript: All authors. \\ Correspondence to: Jai-Kyong Pyon, MD, PhD. Professor, Department of Plastic Surgery, Samsung Medical Center, Sungkyunkwan University School \\ of Medicine, 81 Irwon-ro, Gangnam, Seoul, Republic of Korea. Email: pspriest.pyon@samsung.com.
}

Background: Contralateral augmentation mammoplasty in implant-based reconstruction could potentially lead to deterioration of the thickness of the mastectomy skin flap and increase postoperative complications of the reconstructed breast. We compared the complication rates of the reconstructed breast in the augmentation and no-augmentation groups among patients undergoing tissue expander/implant breast reconstruction.

Methods: Patients who underwent mastectomy followed by tissue expander/implant breast reconstruction between February 2010 and April 2018 were retrospectively reviewed. The primary outcome measures were complications and the need for a revision operation. The augmentation and no-augmentation groups underwent propensity score-matched analysis and the matched cases underwent multivariable logistic regression analysis.

Results: From the 234 patients in the augmentation group and 517 patients in the no-augmentation group, 200 propensity score-matched pairs were obtained. Analysis of the matched pairs revealed that the augmentation group as compared to the no-augmentation group showed a significantly higher overall complication rate $(13.5$ percent versus 6.5 percent; $\mathrm{P}=0.025)$ and revision operation rate $(9.0$ percent versus 3.0 percent; $\mathrm{P}=0.019)$. Multivariable conditional logistic regression analyses of the matched cases revealed that contralateral augmentation (odds ratio, 3.457; 95\% confidence interval, 1.039-11.498; $\mathrm{P}=0.043$ ) was associated with increased odds for a revision operation of the reconstructed breast.

Conclusions: This study investigated the postoperative complications of the reconstructed breast associated with contralateral augmentation mammoplasty in patients who underwent mastectomy followed by tissue expander/implant breast reconstruction. The augmentation group had a higher revision operation rate than did the no-augmentation group. A clinical evaluation of the risks and benefits of contralateral augmentation and preoperative counseling may be indicated for patients who are undergoing implant-based breast reconstruction and are candidates for contralateral augmentation mammoplasty.

Keywords: Two-stage breast reconstruction; implant reconstruction; complication; matching procedure; augmentation mammoplasty

Submitted May 19, 2020. Accepted for publication Sep 03, 2020.

doi: $10.21037 /$ gs-20-509

View this article at: http://dx.doi.org/10.21037/gs-20-509 


\section{Introduction}

After the first radical mastectomy was performed by Halsted in 1882 (1), extirpative procedures for breast cancer have evolved toward less radical resection while maintaining maximum survival. Modified radical mastectomy, which preserves the pectoralis muscles, was first reported by Patey and Dyson in 1948 (2) and became the standard breast cancer operation based on the evidence of equivalent oncologic outcomes $(3,4)$. Currently, skin-sparing mastectomy $(5,6)$, which was gradually introduced in the 1990s for immediate breast reconstruction (7), and the more conservative nipple-sparing mastectomy are widely performed on the basis of the evidence of their oncologic and surgical safety as well as their superior cosmetic outcomes $(8,9)$. The incidence of breast reconstruction surgery after mastectomy has increased due to advances in the field, with two-stage tissue expander/implant reconstruction being the most common reconstruction method currently used (10).

A recent trend in breast reconstruction is performing a contralateral matching procedure, including breast reduction, augmentation, and mastopexy, on the contralateral breast. This became an integral part of the care of breast cancer patients in less than two decades (11). Several studies evaluated the effect of a matching procedure on the postoperative complications of breast reconstruction and found that a matching procedure can be safely performed with breast reconstruction (11-18). However, most of those studies assessed the outcomes of autologous breast reconstruction only $(12,13)$ or grouped different matching procedures into a single variable and evaluated the surgical outcomes as those from a single procedure rather than the outcomes of each matching procedure separately (15-17). However, the effect of each matching procedure on the surgical outcomes of breast reconstruction can be different because contralateral augmentation causes the volume of the reconstructed breast to increase, while contralateral reduction causes the volume of the reconstructed breast to decrease. Regarding the reconstruction method, implant-based reconstruction can compromise blood supply to the mastectomy skin flap, and serial inflation of the tissue expander can cause thinning of the mastectomy skin flap.

A decrease in skin flap thickness was demonstrated to be associated with postoperative complications after mastectomy (19-23). Among the combinations of reconstruction methods and contralateral matching procedures, both procedures of the implant-based reconstruction and contralateral augmentation combination have the potential to lead to deterioration of the thickness and circulation of the mastectomy skin flap. Consequently, postoperative complications to increase. In implantbased reconstruction, the skin envelope used to cover the implant is usually limited to the remnant skin flap left from the mastectomy. It is thus difficult to supply a sufficient skin envelope from adjacent or remote soft tissue in such cases, unlike in an autologous reconstruction. In addition, in patients undergoing contralateral augmentation, the demand for a skin envelope to cover a reconstructed breast mound that is larger than the original breast is increased. We thus hypothesized that the potential lack of a sufficient skin envelope for the reconstructed breast, in combination with the inflation of the tissue expander to reach the size of the contralateral breast (which is indicated for contralateral augmentation), may be associated with postoperative complications of the reconstructed breast. We present the following article in accordance with the STROBE reporting checklist (available at http://dx.doi.org/10.21037/gs-20509).

\section{Methods}

\section{Study design and patients}

Prospectively recorded data from consecutive patients who underwent mastectomy followed by tissue expander/implant breast reconstruction at our institution between February 2010 and April 2018 were retrospectively reviewed. This study was approved by the Institutional Review Board of Samsung Medical Center (IRB No.: 2020-01-094), and was performed in accordance with the principles of the Declaration of Helsinki (as revised in 2013). The requirement of obtaining individual consent was waived for this retrospective analysis. We included all patients who underwent the exchange of the expander to the permanent implant as the second stage of a unilateral tissue expander/ implant breast reconstruction and were followed up at least 6 months postoperatively. Patients who had a contralateral reduction mammoplasty simultaneously with the unilateral breast reconstruction or had a previous breast procedure, including augmentation or reduction mammoplasty, were excluded from this study. Patients who had delayed reconstruction were also excluded because the amount and characteristics of a remnant mastectomy skin flap are different than those seen in an immediate reconstruction. 


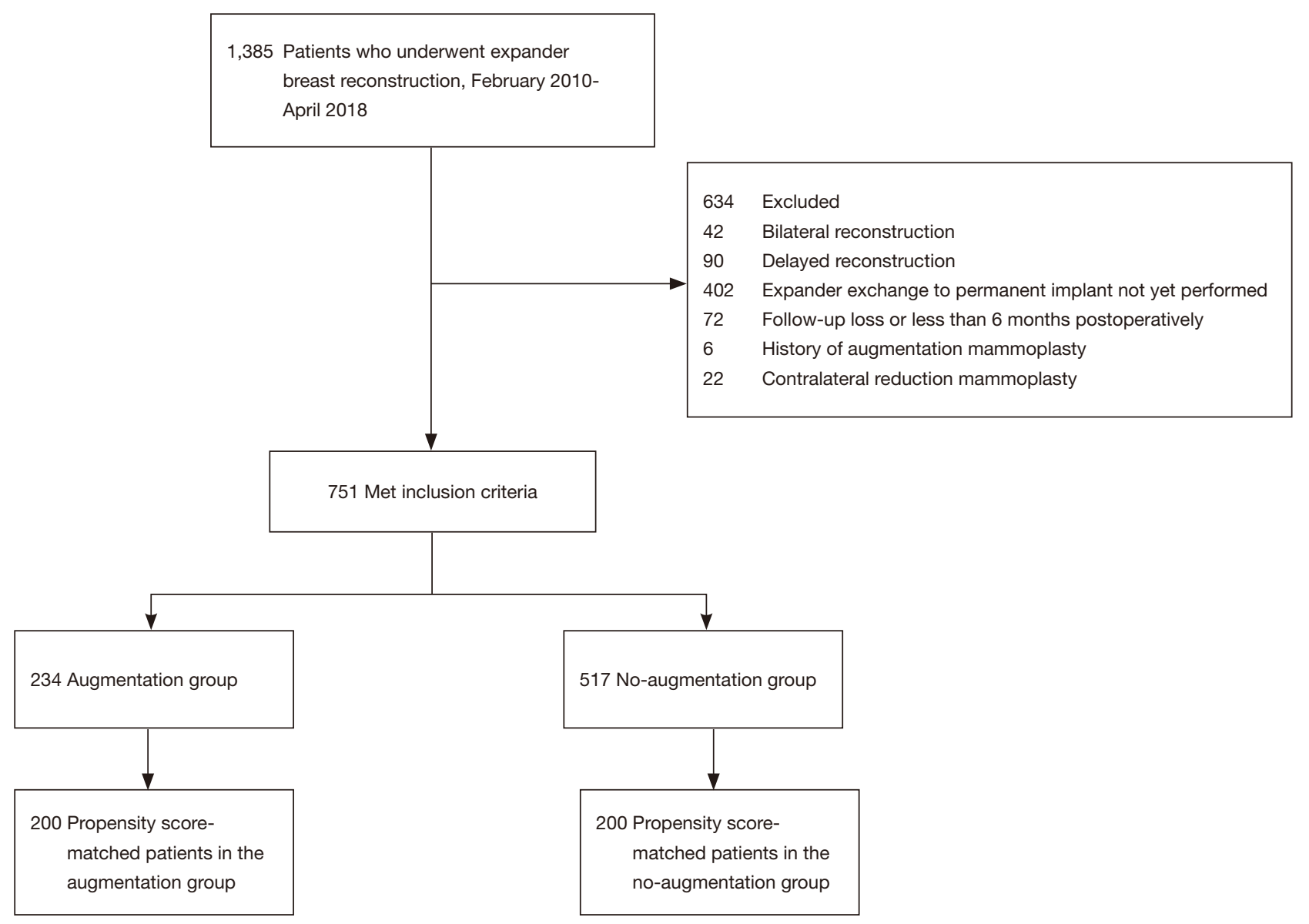

Figure 1 Patient selection process.

The final cohort consisted of 751 patients (Figure 1). Reconstructions were performed by one of the four senior surgeons (BJJ, JKP, GHM, and SIB).

\section{Surgical technique}

After mastectomy, a tissue expander was placed in the subpectoral space. The tissue expander was chosen based on breast width, height, and mastectomy weight. For patients who wanted contralateral augmentation, an expander with a width larger than that of the original breast was chosen. Use of an acellular dermal matrix (ADM) was determined based on the following: the attending surgeons' decision, a need for infero-lateral implant coverage, and a thorough discussion with patients considering their desire and financial burden. Inflation of the expander was started four weeks postoperatively, and an average of $100 \mathrm{~mL}$ of saline $(30-150 \mathrm{~mL})$ was injected at each visit every three to four weeks at an outpatient clinic. The exchange of the expander with a permanent implant was usually performed six months after placement of the expander. All of the contralateral augmentation procedures included in this study were performed simultaneously with the exchange of the expander with a permanent implant. For contralateral augmentation, inframammary incisions were used, and the implant was inserted in the sub-pectoral spaces. The implant size of the augmentation side was decided before surgery based on patients' desire and clinical examination of the mastectomy skin flap by the attending surgeons.

\section{Outcome measurement}

Patients were divided into two cohorts, augmentation and no-augmentation, depending on whether contralateral augmentation mammoplasty was performed simultaneously with the two-stage reconstruction. The clinical and surgical 
variables of the second stage of the operation, i.e., implant placement, that were retrieved included age, body mass index (BMI), smoking history, medical comorbidities (diabetes and hypertension), history of radiotherapy and chemotherapy, implant type and size, and operating surgeon. History of chemotherapy was defined as previously undergoing neoadjuvant and/or adjuvant chemotherapy. History of radiotherapy was defined as undergoing radiotherapy before or after the mastectomy and before the second stage of reconstruction. Operative variables of the first stage of the operation, i.e., tissue expander placement, including mastectomy type (skin- or nipple-sparing), mastectomy weight, ADM use, and tissue expander size, were also analyzed.

Patients in the two cohorts were matched for clinical and operative variables, including age, BMI, smoking history, diabetes, hypertension, history of radiotherapy, history of chemotherapy, mastectomy type, mastectomy weight, $\mathrm{ADM}$ use, implant type, and operating surgeon. Outcome variables were complications and revision operation rates of the reconstructed breasts. Complications included infection, wound dehiscence, seroma/hematoma, capsular contracture, implant malpositioning, and implant rupture. Wound dehiscence was defined as wound breakdown along the margin of the surgical incision $(\geq 0.5 \mathrm{~cm})$ that was treated conservatively or with surgical intervention. Seroma/ hematoma was defined as an abnormal collection of fluid or blood, which was treated by surgical intervention. Capsular contracture was contracture diagnosed as Baker class III or IV by the attending surgeon. Implant malpositioning was defined as an implant that needed additional operative procedures to correct its dislocation or malrotation. Implant rupture was defined as a tear in the outer shell of the implant diagnosed using magnetic resonance imaging.

\section{Statistical analysis}

Continuous variables are presented as mean \pm standard deviation, and categorical variables are summarized as frequency and percentage. Before matching patients in the two groups, clinical and operative categorical variables of the groups were compared using a $\chi^{2}$ test or Fisher's exact test and continuous variables were compared using an independent $t$-test. Propensity score-matching was used to reduce the selection bias for the group variable. Logistic regression was used to estimate the propensity score, where the augmentation group was regressed on baseline characteristics and one-to-one nearest-neighbor matching was performed with a 0.2 caliper width of the pooled standard deviation of the propensity score logit (24). We verified the balance across groups in the matched sample using the standardized mean difference $(<0.1$ in absolute value). A generalized estimating equation and Fisher's exact test were used to evaluate complication and revision operation rates for the matched data. To identify potentially relevant factors associated with the outcome variables, multivariable conditional logistic regression analyses were performed using the matched cases. Adjusted odds ratios are reported with $95 \%$ confidence intervals (CIs). Statistical significance was defined as $\mathrm{P}<0.05$. All analyses were performed using SAS version 9.4 (SAS Institute, Cary, NC, USA).

\section{Results}

We evaluated 751 cases of mastectomy followed by tissue expander/implant breast reconstruction, which included 234 cases in the augmentation group and 517 cases in the no-augmentation group. Except for mastopexy which was performed on the contralateral breast for 28 patients, no procedure was performed in the contralateral breast of patients in the no-augmentation group. The mean age of all patients was $43.6 \pm 7.4$ years (range, 18-66 years), the mean BMI was $22.0 \pm 2.7 \mathrm{~kg} / \mathrm{m}^{2}$ (range, $15.1-33.7 \mathrm{~kg} / \mathrm{m}^{2}$ ), and the mean mastectomy weight was $351.4 \pm 167.8 \mathrm{~g}$ (range, $40-1,289 \mathrm{~g}$ ). The average follow-up was 25.2 months (range, 6.0-113.9 months) after the second-stage operation. Patients in the augmentation group were significantly younger $(42.0 \pm 6.6$ vs. $44.3 \pm 7.6$ years old, $\mathrm{P}<0.001)$ and had a lower BMI $\left(21.0 \pm 2.3\right.$ vs. $\left.22.5 \pm 2.8 \mathrm{~kg} / \mathrm{m}^{2}, \mathrm{P}<0.001\right)$ than those in the no-augmentation group. Mastectomy weight $(259.3 \pm 115.7$ vs. $393.1 \pm 171.1 \mathrm{~g}, \mathrm{P}<0.001)$ and tissue expander size $(354.2 \pm 67.1$ vs. $402.5 \pm 96.4 \mathrm{cc}, \mathrm{P}<0.001)$ were significantly lower in the augmentation group than in the no-augmentation group, while implant size for reconstruction was significantly greater in the augmentation group than in the no-augmentation group $(359.9 \pm 74.7 \mathrm{vs}$. $308.4 \pm 111.3 \mathrm{cc}, \mathrm{P}<0.001)$. The mean volume of implant for augmentation were $182.9 \pm 37.2 \mathrm{cc}$ (range, 90-410 cc). All additional clinical and operative characteristics are summarized in Table 1. After performing propensity score one-to-one matching, 200 patients in each group were selected and all matching variables were well matched using standardized mean differences that were $<10$ percent. Tissue expander size was greater in the augmentation group than in the no-augmentation group, but the difference 
Table 1 Baseline characteristics of all patients

\begin{tabular}{|c|c|c|c|c|}
\hline Characteristic & All patients (\%) & Augmentation group (\%) & No augmentation group (\%) & $\mathrm{P}$ \\
\hline \multicolumn{5}{|l|}{ Patient demographics } \\
\hline Age, yr & $43.6 \pm 7.4$ & $42.0 \pm 6.6$ & $44.3 \pm 7.6$ & $<0.001^{*}$ \\
\hline BMI, kg/m² & $22.0 \pm 2.7$ & $21.0 \pm 2.3$ & $22.5 \pm 2.8$ & $<0.001^{*}$ \\
\hline Diabetes & $10(1.3)$ & $5(2.1)$ & $5(1.0)$ & 0.300 \\
\hline Hypertension & $36(4.8)$ & $6(2.6)$ & $30(5.8)$ & 0.082 \\
\hline Radiotherapy & $92(12.3)$ & $27(11.5)$ & $65(12.6)$ & 0.779 \\
\hline Chemotherapy & $301(40.1)$ & $89(38.0)$ & $212(41.0)$ & 0.491 \\
\hline Skin-sparing & $551(73.4)$ & $164(70.1)$ & $387(74.9)$ & \\
\hline Nipple-sparing & $200(26.6)$ & $70(29.9)$ & $130(25.1)$ & \\
\hline Mastectomy weight (g) & $351.4 \pm 167.8$ & $259.3 \pm 115.7$ & $393.1 \pm 171.1$ & $<0.001^{*}$ \\
\hline ADM use & $453(60.3)$ & $139(59.4)$ & $314(60.7)$ & 0.791 \\
\hline Tissue expander size (cc) & $387.4 \pm 91.0$ & $354.2 \pm 67.1$ & $402.5 \pm 96.4$ & $<0.001^{*}$ \\
\hline Implant size for reconstruction (cc) & $324.5 \pm 104.1$ & $359.9 \pm 74.7$ & $308.4 \pm 111.3$ & $<0.001^{*}$ \\
\hline Implant type & & & & 0.549 \\
\hline Smooth & $325(43.3)$ & $97(41.5)$ & $228(44.1)$ & \\
\hline Surgeon 3 & $238(31.7)$ & $75(32.1)$ & $163(31.5)$ & \\
\hline Surgeon 4 & $200(26.6)$ & $43(18.4)$ & $157(30.4)$ & \\
\hline
\end{tabular}

*, statistically significant. BMI, body mass index; ADM, acellular dermal matrix.

was not statistically significant $(358.4 \pm 69.8 v$ vs. $349.5 \pm 64.0$ cc, $\mathrm{P}=0.107)$, and implant size for reconstruction was significantly greater in the augmentation group than in the no-augmentation group $(369.7 \pm 72.7$ vs. $251.1 \pm 91.0 \mathrm{cc}$, $\mathrm{P}<0.001)$. The mean volume of the implant for augmentation was $183.1 \pm 35.0 \mathrm{cc}$ (range, 90-295 cc) (Table 2).

For unmatched cases, the complication rate of the reconstructed breast was significantly higher for the augmentation group than for the no-augmentation group (13.2 percent versus 6.4 percent; $\mathrm{P}=0.003$ ). Among the complications, wound dehiscence was significantly greater in the augmentation group than in the no-augmentation group (3.0 percent versus 0.8 percent; $\mathrm{P}=0.042$ ). More revision operations were performed in the augmentation group than in the no-augmentation group (9.0 percent versus 3.1 percent; $\mathrm{P}=0.001)$. For the matched cases, the complication rate of the reconstructed breast was significantly greater in the augmentation group than in the no-augmentation group (13.5 percent versus 6.5 percent; $\mathrm{P}=0.025$ ). However, no specific complications were significantly different between 
Table 2 Baseline characteristics of the propensity score one-to-one matched patients

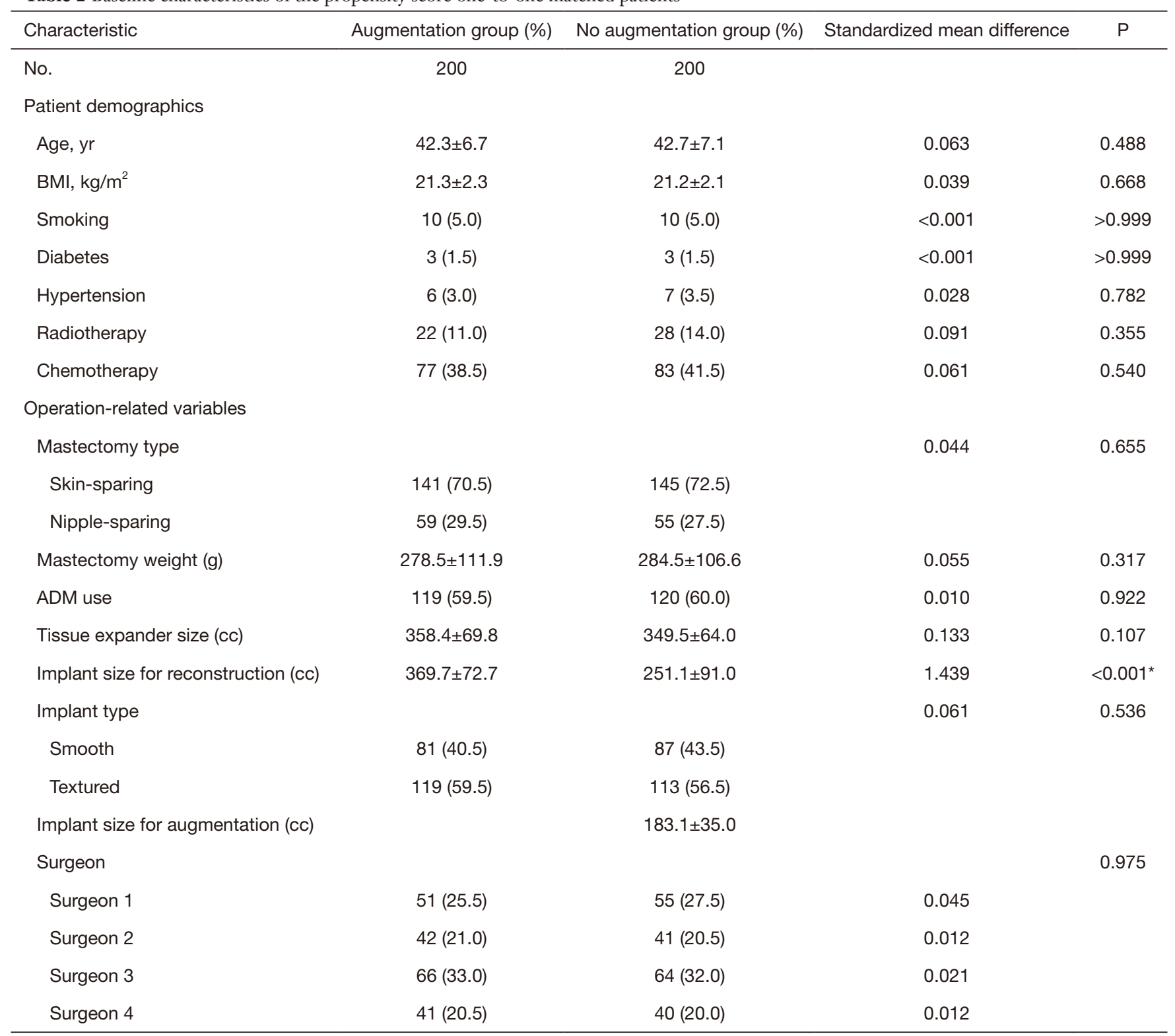

*, statistically significant. BMI, body mass index; ADM, acellular dermal matrix.

the two groups. More revision operations were performed in the augmentation group than in the no-augmentation group, with statistical significance (9.0 percent versus 3.0 percent; $\mathrm{P}=0.019$ ) (Table 3).

After controlling for potential confounders using multivariable conditional logistic regression analysis of the matched cases, contralateral augmentation mammoplasty (OR, 3.457; 95\% CI, 1.039-11.498; $\mathrm{P}=0.043)$ was associated with significantly increased odds for a revision operation of the reconstructed breast after the second stage of tissue expander/implant breast reconstruction (Table 4). Conversely, no significant factors were associated with overall complications in the multivariable analysis performed using matched data (Table 5).

\section{Discussion}

This study evaluated the effect of contralateral augmentation 
Table 3 Postoperative outcomes of all patients and propensity score-matched patients

\begin{tabular}{|c|c|c|c|c|c|c|}
\hline Variable & \multicolumn{3}{|c|}{ All patients $(n=751)$} & \multicolumn{3}{|c|}{ Propensity score-matched patients $(n=400)$} \\
\hline No. & 234 & 517 & & 200 & 200 & \\
\hline Complications & $31(13.2)$ & $33(6.4)$ & $0.003^{*}$ & $27(13.5)$ & $13(6.5)$ & $0.025^{*}$ \\
\hline Infection & $1(0.4)$ & $3(0.6)$ & $>0.999$ & $1(0.5)$ & $2(1.0)$ & 0.571 \\
\hline Seroma/hematoma & $1(0.4)$ & $3(0.6)$ & $>0.999$ & $1(0.5)$ & 0 & $>0.999$ \\
\hline Capsular contracture & $10(4.3)$ & $14(2.7)$ & 0.365 & $9(4.5)$ & $6(3.0)$ & 0.441 \\
\hline Implant malpositioning & $5(2.1)$ & $2(0.4)$ & 0.057 & $5(2.5)$ & $1(0.5)$ & 0.140 \\
\hline Implant rupture & $9(3.8)$ & $9(1.7)$ & 0.136 & $8(4.0)$ & $4(2.0)$ & 0.379 \\
\hline
\end{tabular}

${ }^{*}$, statistically significant.

Table 4 Multivariable conditional logistic regression analysis of revision operation for matched cases

\begin{tabular}{lcc}
\hline Variable & Adjusted OR $(95 \% \mathrm{Cl})$ & $\mathrm{P}$ \\
\hline Contralateral augmentation & $3.457(1.039-11.498)$ & $0.043^{\star}$ \\
Tissue expander size & $1.002(0.995-1.010)$ & 0.509 \\
Implant size & $0.999(0.993-1.006)$ & 0.799 \\
\hline
\end{tabular}

*, statistically significant. $\mathrm{Cl}$, confidence interval.

Table 5 Multivariable conditional logistic regression analysis of overall complications for matched cases

\begin{tabular}{lcc}
\hline Variable & Adjusted OR $(95 \% \mathrm{Cl})$ & $\mathrm{P}$ \\
\hline Contralateral augmentation & $2.279(0.934-5.560)$ & 0.070 \\
Tissue expander size & $1.0020 .997-1.008)$ & 0.432 \\
Implant size & $1.000(0.995-1.005)$ & 0.906 \\
\hline
\end{tabular}

*, statistically significant. $\mathrm{Cl}$, confidence interval.

mammoplasty on the postoperative complications of tissue expander/implant breast reconstruction and demonstrated that contralateral augmentation was significantly associated with the need for a revision operation on the reconstructed breast after exchanging the expander for a permanent implant. Propensity score-matched analysis excluded the effects of clinical and operative variables, including age, BMI, smoking history, diabetes, hypertension, history of radiotherapy, history of chemotherapy, mastectomy type, mastectomy weight, ADM use, implant type, and operating surgeon, that were different between the augmentation and no-augmentation groups and had the potential to affect the surgical outcomes. To the best of our knowledge, this study is the largest clinical series that evaluated the impact of contralateral augmentation mammoplasty as a matching procedure on the postoperative complications of the reconstructed breast after implant-based breast reconstruction.

In their analysis of a national multi-institutional database of 24,191 patients, Cooney et al. found that a matching procedure performed simultaneously with an immediate breast reconstruction was not significantly associated with postoperative complications (17). Their study was significant for its assessment of the general surgical risk associated with performing a matching procedure simultaneously with breast reconstruction in a large population. However, Cooney et al. did not assess the effects of the matching procedures of augmentation, reduction, and mastopexy separately on a specific reconstruction procedure such as autologous or implant-based breast reconstruction. Liu et al. assessed the surgical outcomes of tissue expander/ implant breast reconstruction combined with contralateral breast augmentation in patients with small breasts and insisted that contralateral augmentation can be safely performed simultaneously with implant-based breast reconstruction (18). However, their evidence is weak because they assessed the surgical outcomes of only 30 patients and there was no control group with which to compare surgical outcomes. Thus, we performed a propensity score-matched analysis 
of the data of 751 patients that included 234 augmentation cases to evaluate the effects of contralateral augmentation mammoplasty on the postoperative complications of tissue expander/implant breast reconstruction. We hypothesized that contralateral augmentation mammoplasty can cause a lack of skin envelope on the reconstruction side to cover an implant larger in size than the original breast. We evaluated the outcomes only after exchanging the expander for the permanent implant because analysis of surgical outcomes after expander placement and expander exchange can mask the surgical risk of contralateral matching procedures. Complications during expander placement occurred at a higher rate than during expander exchange $(25,26)$, so the risk associated with contralateral matching procedures could be underestimated if the surgical outcomes after expander placement are included.

Several studies have evaluated factors relevant to ischemic complications associated with a mastectomy skin flap, such as mastectomy skin flap necrosis, after breast reconstruction. Established factors include a history of smoking, obesity, mastectomy specimen weight, and radiotherapy (27-34). Anatomic factors such as skin flap thickness could potentially be associated with ischemic complications but they have rarely been studied. Frey et al. assessed the association between skin flap thickness in nipple-sparing mastectomy and ischemic complications after breast reconstruction using magnetic resonance imaging and found that a postoperative skin flap thickness of $<8.0 \mathrm{~mm}$ was an independent predictor of ischemic complications (21). In our study, we hypothesized that the additional inflation volume of the tissue expander for contralateral augmentation mammoplasty can cause the mastectomy skin flap to become thinner, which will eventually increase postoperative complications for the reconstructed breast. However, we cannot confirm our hypothesis because this retrospective study did not include quantitative and objective analyses of mastectomy skin flap thickness. Further studies that include objective measurements of mastectomy skin flap thickness may strengthen the findings of our study and help determine the acceptable amount of inflation volume for contralateral augmentation.

Contralateral augmentation mammoplasty can be performed with breast reconstruction either to address a need for symmetry or the wish of the patient. Implantbased breast reconstruction can result in a non-ptotic breast with a contour that does not match that of a natural breast, especially if that breast has some components of ptosis (14). In this case, contralateral augmentation mammoplasty can be used to improve symmetry and aesthetic outcome. Contralateral augmentation can also be performed when patients want larger breasts because of dissatisfaction with their body image or concern about the importance of the breast in social and romantic relationships $(35,36)$. Several studies have demonstrated that improved symmetry $(12,37,38)$ and patient satisfaction, including psychosocial and sexual well-being $(12,13,18)$, can be obtained when contralateral augmentation mammoplasty is performed in conjunction with breast reconstruction. In addition, a retrospective study by Razdan et al. on 553 patients who underwent unilateral two-stage tissue expander/implant breast reconstruction demonstrated that contralateral augmentation yielded greater patient satisfaction with the outcome than did contralateral reduction mammoplasty and mastopexy (39). These studies have suggested that contralateral augmentation mammoplasty can provide the obvious benefits of a good aesthetic outcome and positive psychological well-being in patients who undergo breast reconstruction. However, the results of our study indicated that contralateral augmentation mammoplasty significantly increases the risk of the need for a revision operation after implant-based reconstruction. In addition to the benefits of contralateral augmentation, the associated risks need to be carefully assessed in preoperative planning and should be addressed during patient counseling.

The present study has some limitations. First, we did not exclude contralateral mastopexy cases because we assumed that the effect of contralateral mastopexy on mastectomy skin flap thickness and postoperative complications in breast reconstruction is minimal, but this assumption was not validated. Second, the patients included in this study were Asian women who had relatively low BMI and small breasts. Studies on other races and ethnicities are warranted to increase the generalizability of the results of this study. Third, delayed breast reconstruction cases were not included because the number of patients at our institution who underwent delayed breast reconstruction and contralateral augmentation mammoplasty was too small. We believe that delayed reconstruction needs to be evaluated separately from immediate reconstruction because the amount of remnant skin flap for delayed reconstruction after mastectomy is usually less than that for immediate reconstruction, and the time between radiotherapy and delayed breast reconstruction differs from that in immediate reconstruction. Therefore, the effects of contralateral augmentation mammoplasty on surgical outcomes can differ from those found in this study, which 
was conducted on immediate reconstruction patients only. Fourth, capsular contracture and implant rupture may not have been fully evaluated in the study population because these complications usually occur several years after surgery. Finally, we could not assess the factors associated with the higher revision operation rate in the augmentation group because there were relatively few patients who underwent a revision operation after exchange of the expander for the permanent implant. Thus, this study could not evaluate specific indications for or against contralateral augmentation mammoplasty with implant-based breast reconstruction. Larger studies such as multicenter or a population-based studies would be required to elucidate the factors associated with the higher revision operation rate in the augmentation group found in the current study.

\section{Conclusions}

The results of this study indicate that contralateral augmentation mammoplasty is associated with significantly increased odds for a revision operation after the second stage of an immediate tissue expander/implant breast reconstruction. Risk-benefit assessment and preoperative counseling are necessary for patients undergoing contralateral augmentation mammoplasty with implantbased breast reconstruction.

\section{Acknowledgments}

We would like to thank Editage (www.editage.co.kr) for English language editing.

Funding: None.

\section{Footnote}

Reporting Checklist: The authors have completed the STROBE reporting checklist. Available at http://dx.doi. org/10.21037/gs-20-509

Data Sharing Statement: Available at http://dx.doi. org/10.21037/gs-20-509

Peer Review File: Available at http://dx.doi.org/10.21037/gs20-509

Conflicts of Interest: All authors have completed the ICMJE uniform disclosure form (available at http://dx.doi. org/10.21037/gs-20-509). The authors have no conflicts of interest to declare.

Ethical Statement: The authors are accountable for all aspects of the work in ensuring that questions related to the accuracy or integrity of any part of the work are appropriately investigated and resolved. This study was approved by the Institutional Review Board of Samsung Medical Center (IRB No.: 2020-01-094), and was performed in accordance with the principles of the Declaration of Helsinki (as revised in 2013). The requirement of obtaining individual consent was waived for this retrospective analysis.

Open Access Statement: This is an Open Access article distributed in accordance with the Creative Commons Attribution-NonCommercial-NoDerivs 4.0 International License (CC BY-NC-ND 4.0), which permits the noncommercial replication and distribution of the article with the strict proviso that no changes or edits are made and the original work is properly cited (including links to both the formal publication through the relevant DOI and the license). See: https://creativecommons.org/licenses/by-nc-nd/4.0/.

\section{References}

1. Loukas M, Tubbs RS, Mirzayan N, et al. The history of mastectomy. Am Surg 2011;77:566-71.

2. Patey DH, Dyson WH. The prognosis of carcinoma of the breast in relation to the type of operation performed. Br J Cancer 1948;2:7-13.

3. Turner L, Swindell R, Bell WG, et al. Radical versus modified radical mastectomy for breast cancer. Ann R Coll Surg Engl 1981;63:239-43.

4. Maddox WA, Carpenter JT Jr, Laws HL, et al. A randomized prospective trial of radical (Halsted) mastectomy versus modified radical mastectomy in 311 breast cancer patients. Ann Surg 1983;198:207-12.

5. Toth BA, Lappert P. Modified skin incisions for mastectomy: the need for plastic surgical input in preoperative planning. Plast Reconstr Surg 1991;87:1048-53.

6. Kroll SS, Ames F, Singletary SE, et al. The oncologic risks of skin preservation at mastectomy when combined with immediate reconstruction of the breast. Surg Gynecol Obstet 1991;172:17-20.

7. Leone MS, Priano V, Franchelli S, et al. Factors affecting symmetrization of the contralateral breast: a 7-year unilateral postmastectomy breast reconstruction experience. Aesthetic Plast Surg 2011;35:446-51. 
8. Wu ZY, Kim HJ, Lee JW, et al. Breast Cancer Recurrence in the Nipple-Areola Complex After Nipple-Sparing Mastectomy With Immediate Breast Reconstruction for Invasive Breast Cancer. JAMA Surg 2019;154:1030-7.

9. Gerber B, Krause A, Dieterich M, et al. The oncological safety of skin sparing mastectomy with conservation of the nipple-areola complex and autologous reconstruction: an extended follow-up study. Ann Surg 2009;249:461-8.

10. Ilonzo N, Tsang A, Tsantes $\mathrm{S}$, et al. Breast reconstruction after mastectomy: A ten-year analysis of trends and immediate postoperative outcomes. Breast 2017;32:7-12.

11. Rizki H, Nkonde C, Ching RC, et al. Plastic surgical management of the contralateral breast in post-mastectomy breast reconstruction. Int J Surg 2013;11:767-72.

12. Ulusal BG, Cheng MH, Wei FC. Simultaneous endoscope-assisted contralateral breast augmentation with implants in patients undergoing postmastectomy breast reconstruction with abdominal flaps. Plast Reconstr Surg 2006;118:1293-302.

13. Huang JJ, Chao LF, Wu CW, et al. Simultaneous scarless contralateral breast augmentation during unilateral breast reconstruction using bilateral differentially split DIEP flaps. Plast Reconstr Surg 2011;128:593e-604e.

14. Kropf N, Cordeiro CN, McCarthy CM, et al. Demystifying trans-axillary augmentation/periareolar mastopexy: a novel, two-stage, single-operation approach to management of the contralateral breast in implant reconstruction. J Plast Reconstr Aesthet Surg 2011;64:485-93.

15. Smith ML, Clarke-Pearson EM, Vornovitsky M, et al. The efficacy of simultaneous breast reconstruction and contralateral balancing procedures in reducing the need for second stage operations. Arch Plast Surg 2014;41:535-41.

16. Salgarello M, Visconti G, Barone-Adesi L, et al. Contralateral breast symmetrisation in immediate prosthetic breast reconstruction after unilateral nipplesparing mastectomy: the tailored reduction/augmentation mammaplasty. Arch Plast Surg 2015;42:302-8.

17. Cooney CM, Sebai ME, Ogbuagu O, et al. Matching Procedures at the Time of Immediate Breast Reconstruction: An American College of Surgeons National Surgical Quality Improvement Program Study of 24,191 Patients. Plast Reconstr Surg 2016;138:959e-68e.

18. Liu HH, Chiang IH, Wang CH, et al. Postmastectomy Breast Reconstruction Combined With Contralateral Breast Augmentation for Taiwanese Women With Small
Breasts. Ann Plast Surg 2017;78:S102-7.

19. Robertson SA, Rusby JE, Cutress RI. Determinants of optimal mastectomy skin flap thickness. Br J Surg 2014;101:899-911.

20. Ashikari AY, Kelemen PR, Tastan B, et al. Nipple sparing mastectomy techniques: a literature review and an inframammary technique. Gland Surg 2018;7:273-87.

21. Frey JD, Salibian AA, Choi M, et al. Mastectomy Flap Thickness and Complications in Nipple-Sparing Mastectomy: Objective Evaluation using Magnetic Resonance Imaging. Plast Reconstr Surg Glob Open 2017;5:e1439.

22. Frey JD, Salibian AA, Choi M, et al. Optimizing Outcomes in Nipple-sparing Mastectomy: Mastectomy Flap Thickness Is Not One Size Fits All. Plast Reconstr Surg Glob Open 2019;7:e2103.

23. Algaithy ZK, Petit JY, Lohsiriwat V, et al. Nipple sparing mastectomy: can we predict the factors predisposing to necrosis? Eur J Surg Oncol 2012;38:125-9.

24. Austin PC. A comparison of 12 algorithms for matching on the propensity score. Stat Med 2014;33:1057-69.

25. Cordeiro PG, McCarthy CM. A single surgeon's 12year experience with tissue expander/implant breast reconstruction: part I. A prospective analysis of early complications. Plast Reconstr Surg 2006;118:825-31.

26. Lovecchio F, Jordan SW, Lim S, et al. Risk Factors for Complications Differ Between Stages of Tissue-Expander Breast Reconstruction. Ann Plast Surg 2015;75:275-80.

27. Robertson SA, Jeevaratnam JA, Agrawal A, et al. Mastectomy skin flap necrosis: challenges and solutions. Breast Cancer (Dove Med Press) 2017;9:141-52.

28. Kanuri A, Liu AS, Guo L. Whom should we SPY? A cost analysis of laser-assisted indocyanine green angiography in prevention of mastectomy skin flap necrosis during prosthesis-based breast reconstruction. Plast Reconstr Surg 2014;133:448e-54e.

29. Lee TJ, Oh TS, Kim EK, et al. Risk factors of mastectomy skin flap necrosis in immediate breast reconstruction using low abdominal flaps. J Plast Surg Hand Surg 2016;50:302-6.

30. Reintgen C, Leavitt A, Pace E, et al. Risk Factor Analysis for Mastectomy Skin Flap Necrosis: Implications for Intraoperative Vascular Analysis. Ann Plast Surg 2016;76 Suppl 4:S336-9.

31. Sue GR, Long C, Lee GK. Management of Mastectomy Skin Necrosis in Implant Based Breast Reconstruction. Ann Plast Surg 2017;78:S208-11.

32. Sue GR, Sun BJ, Lee GK. Complications After Two- 
Stage Expander Implant Breast Reconstruction Requiring Reoperation: A Critical Analysis of Outcomes. Ann Plast Surg 2018;80:S292-4.

33. Ito H, Ueno T, Suga H, et al. Risk Factors for Skin Flap Necrosis in Breast Cancer Patients Treated with Mastectomy Followed by Immediate Breast Reconstruction. World J Surg 2019;43:846-52.

34. Rudolph M, Moore C, Pestana IA. Operative risk stratification in the obese female undergoing implantbased breast reconstruction. Breast J 2019;25:1182-6.

35. Schain WS, Jacobs E, Wellisch DK. Psychosocial issues in breast reconstruction. Intrapsychic, interpersonal, and practical concerns. Clin Plast Surg 1984;11:237-51.

36. Didie ER, Sarwer DB. Factors that influence the decision to undergo cosmetic breast augmentation surgery. J Womens Health (Larchmt) 2003;12:241-53.

Cite this article as: Park JW, Kim S, Jeon BJ, Mun GH, Bang SI, Pyon JK. Effect of contralateral augmentation on postoperative complications after the second stage of tissue expander/implant breast reconstruction. Gland Surg 2020;9(5):1182-1192. doi: 10.21037/gs-20-509
37. Salgarello M, Visconti G, Barone-Adesi L, et al. Contralateral Breast Symmetrisation in Immediate Prosthetic Breast Reconstruction after Unilateral Nipple-Sparing Mastectomy: The Tailored Reduction/ Augmentation Mammaplasty. Arch Plast Surg 2015;42:302-8.

38. Nava MB, Spano A, Cadenelli P, et al. Extra-projected implants as an alternative surgical model for breast reconstruction. Implantation strategy and early results. Breast 2008;17:361-6.

39. Razdan SN, Panchal H, Albornoz CR, et al. Impact of Contralateral Symmetry Procedures on LongTerm Patient-Reported Outcomes following Unilateral Prosthetic Breast Reconstruction. J Reconstr Microsurg 2019;35:124-8. 